\title{
Empleo del método electroquímico acelerado AC-DC-AC para la predicción del tiempo de vida útil de pinturas base agua
}

\author{
M. Bethencourt", F.J. Botana", L. González-Rovira", M. Marcos" y R.M. Osuna* \\ Resumen En este trabajo se propone un método acelerado de evaluación de metales pintados. \\ Concretamente, el método se ha aplicado al estudio del proceso de degradación de dos \\ pinturas de base agua aplicadas sobre un acero al carbono. El método está basado en la \\ aplicación de ciclos en los que se combinan la medida de espectros de impedancia \\ electroquímica, etapas de polarización catódica y el registro de la evolución del potencial \\ de corrosión del sistema en función del tiempo. Los resultados obtenidos mediante este \\ método se comparan con los obtenidos al estudiar la evolución de los espectros de \\ impedancia de ambos sistemas en función del tiempo de inmersión, para periodos de \\ inmersión del orden de 200 d.
}

Palabras clave Pinturas base agua. Acero pintado. Espectroscopia de impedancia electroquímica. Ensayos acelerados. Deslaminación catódica.

\section{Lifetime prediction of water-borne paints with the AC-DC-AC accelerated electrochemical method}

\begin{abstract}
In this study an accelerated method for the evaluation of painted metals is proposed. Specifically, the proposed method has been applied in a study of the process of degradation of two water-based paints applied to a carbon steel surface. The technique is based on the application of cycles combining measurement of electrochemical impedance spectra, cathodic polarisation steps, and recording of the evolution of the corrosion potential of the system in function of time. The results obtained from this method are compared with those obtained from studying the evolution of the impedance spectra of both systems in function of the time of immersion, for periods of immersion of the order of 200 days.
\end{abstract}

Keywords Water-borne paints. Coated steel. Electrochemical impedance spectroscopy. Accelerated tests. Cathodic delamination.

\section{INTRODUCCIÓN}

La sustitución de los recubrimientos tradicionales de base disolvente orgánico por recubrimientos de alto contenido en sólidos en fase acuosa, es una tendencia bien establecida que se ha implantado paulatinamente a lo largo de los últimos 20 años. La principal fuerza que está llevando el mercado hacia los productos al agua es la mayor presión de los reglamentos a favor de productos con bajo contenido en compuestos orgánicos volátiles (COV), que resultan dañinos para el medio ambiente ${ }^{[1]}$.

Ante la inminente llegada de nuevos productos al mercado de las pinturas, se hacen necesarios métodos que evalúen de una forma rápida la capaci- dad protectora anticorrosiva de los recubrimientos orgánicos, en función del uso que vayan a desarrollar. La clave para que el método se considere práctico se baśa en que la evaluación tenga una buena correlación entre los ensayos de laboratorio y los ensayos de campo, para poder, así, predecir el tiempo de vida útil de la pintura en servicio ${ }^{[2]}$.

Los métodos electroquímicos, principalmente la espectroscopia de impedancia electroquímica (EIS), se han venido empleando con éxito para la evaluación actual y el estudio de los cambios experimentados por las propiedades físicas de diversos tipos de métodos de protección ${ }^{[3-5]}$. Sin embargo, no es posible predecir, a partir del estado anticorrosivo actual, el futuro comportamiento electroquímico

(*) Departamento de Ciencia de los Materiales e Ingeniería Metalúrgica y Química Inorgánica. Universidad de Cádiz. Facultad de Ciencias del Mar y Ambientales. Avda. República Saharaui s/n, 11510 Puerto Real, Cádiz, España.

(**) Dpto. de Ingeniería Mecánica y Diseño Industrial, Escuela Superior de Ingeniería. C) Chile, 1, 11002-Cádiz, España. 
que presentará, por ejemplo, un sistema metalpintura.

En este trabajo se ha empleado, además del registro de EIS a lo largo del tiempo de exposición al medio corrosivo, el método electroquímico acelerado (AC-DC-AC), para predecir la vida útil de pinturas acrílicas de base agua. Éste, consiste en el empleo de la combinación de fases de estrés corrosivo mediante polarización catódica, con evaluaciones mediante EIS y circuito abierto del estado actual de las probetas pintadas. Esta técnica puede llegar a ser una herramienta muy útil para la evaluación rápida y para la clasificación de la actuación de las pinturas con respecto a su comportamiento anticorrosivo. En este sentido, conviene señalar que uno de los trabajos pioneros sobre la técnica fue el publicado por Hollaender ${ }^{[6]}$ en el ámbito de los envases alimenticios. Adicionalmente, en la actualidad, el método AC-DC-AC se está utilizando por otros autores en el campo de las pinturas aplicadas sobre aceros, obteniéndose buenas correlaciones con la técnica EIS en un menor intervalo de tiempo ${ }^{[7-9]}$.

\section{MATERIALES Y PROCEDIMIENTO EXPERIMEN- TAL}

\subsection{Material y preparación de probetas}

Se ha estudiado el comportamiento de dos pinturas acrílicas de base agua. La primera de ellas, Pintura 1, es comercial, Intercryl 506 de pinturas International. La segunda, Pintura 2, es de nueva formulación y está compuesta por una resina acrílica con una concentración en volumen de fosfato de zinc del $17 \%$ sobre pintura seca. Ambas, contienen pigmentos ecológicos como el fosfato de zinc. El material ensayado fue un acero al carbono de baja aleación tipo AISI 1010. Se tomaron probetas rectangulares de $100 \times 150 \times 2 \mathrm{~mm}$ y se sometieron a la preparación superficial tipo cepillado St2 según la norma UNE 48302. Seguidamente, tras desengrasar las muestras con acetona, se procedió a la aplicación de la pintura sobre las probetas hasta alcanzar un espesor de $75 \mathrm{~mm}$, espesor recomendado en la hoja técnica de la primera pintura.

\subsection{Medidas electroquímicas}

La capacidad protectora del recubrimiento se evaluó realizando un seguimiento, mediante técnicas electroquímicas, del comportamiento de las muestras en ensayos de inmersión. Para ello, se construyeron cel- das electroquímicas en las quedaban expuestas 13 $\mathrm{cm}^{2}$ de una de las caras pintadas de las probetas, que actuaban como electrodo de trabajo. Como medio agresivo se utilizaron disoluciones de $\mathrm{NaCl}$ a 3,5 \% (peso). Como electrodo de referencia se utilizó uno de calomelanos saturado y de contraelectrodo una barra de grafito. Para la realización de las medidas electroquímicas se ha empleado un potenciostato 1287 acoplado a un analizador de respuesta en frecuencia (FRA) 1255, ambos de Solartron.

\subsubsection{Espectroscopia de impedancia electroquímica}

Se registraron, a lo largo del tiempo, espectros de impedancia electroquímica en las probetas pintadas hasta que se observó la degradación del sistema por un periodo máximo de $230 \mathrm{~d}$. La técnica EIS permite analizar de forma discriminada la respuesta electroquímica del sistema metal-pintura.

El rango de medida de frecuencia empleado para registrar los espectros de impedancia fue de $10^{5}$ a $10^{-2} \mathrm{~Hz}$ y la amplitud de la señal de $10 \mathrm{mV}$ respecto al potencial de corrosión.

\subsubsection{Técnica acelerada AC-DC-AC}

El procedimiento consta de las siguientes fases:

I) Registro de EIS en el potencial libre de corrosión (OCP). Este, indica el estado actual del sistema metal-pintura.

II) Aplicación de polarización catódica a $-2 \mathrm{~V}$ durante $20 \mathrm{~min}$.

III) Tiempo de relajación durante $180 \mathrm{~min}$, hasta alcanzar de nuevo OCP.

Los pasos I a III se repiten mediante ciclos programados hasta que se observa la pérdida de la capacidad protectora del recubrimiento en el registro de EIS del paso I del ciclo siguiente. El tiempo aproximado que requiere un ciclo es de 3 horas y media.

El potencial aplicado en el paso II y la duración de los pasos II y III pueden variar en función de las características del sistema estudiado. Los valores que se proponen corresponden a aquellos que han conducido a los mejores resultados para el sistema objeto de estudio.

\section{RESULTADOS Y DISCUSIÓN}

Las figuras 1 y 3 muestran los resultados del empleo de la técnica AC-DC-AC y las figuras 2 y 4 , 
la evolución de EIS en el tiempo, para las dos pinturas estudiadas.

En la figura 1 se observa que, tras 4 ciclos de estrés catódico, la Pintura 1 pierde sus propiedades protectoras; esto se aprecia mejor en el descenso del módulo de la impedancia en el diagrama de Bode. Paralelamente, en el diagrama de Nyquist se detecta un considerable aumento del arco durante los primeros ciclos del ensayo. Este comportamiento, en el caso particular de las pinturas de base agua, suele relacionarse con el proceso incompleto de coalescencia durante la etapa de secado ${ }^{[10]}$.

Este mismo efecto se observa en la figura 2 a lo largo del tiempo de inmersión, hasta llegar a los $230 \mathrm{~d}$, período en que la pintura sufre una considerable degradación. De la comparación de ambos diagramas, podemos concluir que la espectroscopia de impedancia electroquímica tras 230 d en inmersión coincide con el espectro después de que la muestra sufre 4 ciclos en el ensayo acelerado AC-DC-AC.

Respecto a la figura 3, es posible observar que, a medida que a la Pintura 2 se le aplican ciclos de estrés catódico, contrariamente a lo que sucedía con la Pintura 1, sufre una degradación paulatina a lo largo del tiempo. Así, después del segundo ciclo, en el diagrama de Bode se comprueba que la pintura acrílica de nueva formulación presenta valores de impedancia menores de $10^{6} \mathrm{~W} \mathrm{~cm}^{2}$, valor éste por el que no puede considerarse que el recubrimiento esté protegiendo adecuadamente a la pintura ${ }^{\left[11^{\text {y }}\right.}{ }^{12]}$. Tras el tercer ciclo, el espectro EIS disminuye más drásticamente y el efecto protector de la pintura se ve afectado considerablemente. Esta evolución se observa mejor en el rango de altas frecuencias de Bode, que se asocia al comportamiento exclusivo de la pintura.

En la figura 4, la evolución del sistema metalpintura es similar a la experimentada por la técnica acelerada. En este caso, en lugar de sufrir una rápida degradación tras un determinado periodo de exposición, como sucedía con la Pintura 1, el recubrimiento va perdiendo progresivamente sus propiedades protectoras y no puede considerarse un momento concreto en que la pintura sufra la degradación final. En este sentido, a pesar de que la pintura contiene fosfato de zinc como pigmento inhibidor, no proporciona una protección pasivante al metal. Por último, si comparamos los espectros de impedancia obtenidos mediante ambas técnicas, es posible considerar que el tercer ciclo del método AC-DC-AC coincide con el espectro correspondiente a los $185 \mathrm{~d}$ de inmersión.

Estos hechos nos proporcionan una primera idea de que acortando el tiempo de evaluación a horas, mediante estrés catódico, obtenemos resultados equivalentes a la técnica EIS en disolución a lo largo de meses de exposición.

Conviene señalar que este método acelerado de aplicación de estrés catódico es posible utilizarlo en recubrimientos que sufran como principal tipo de degradación en servicio la deslaminación
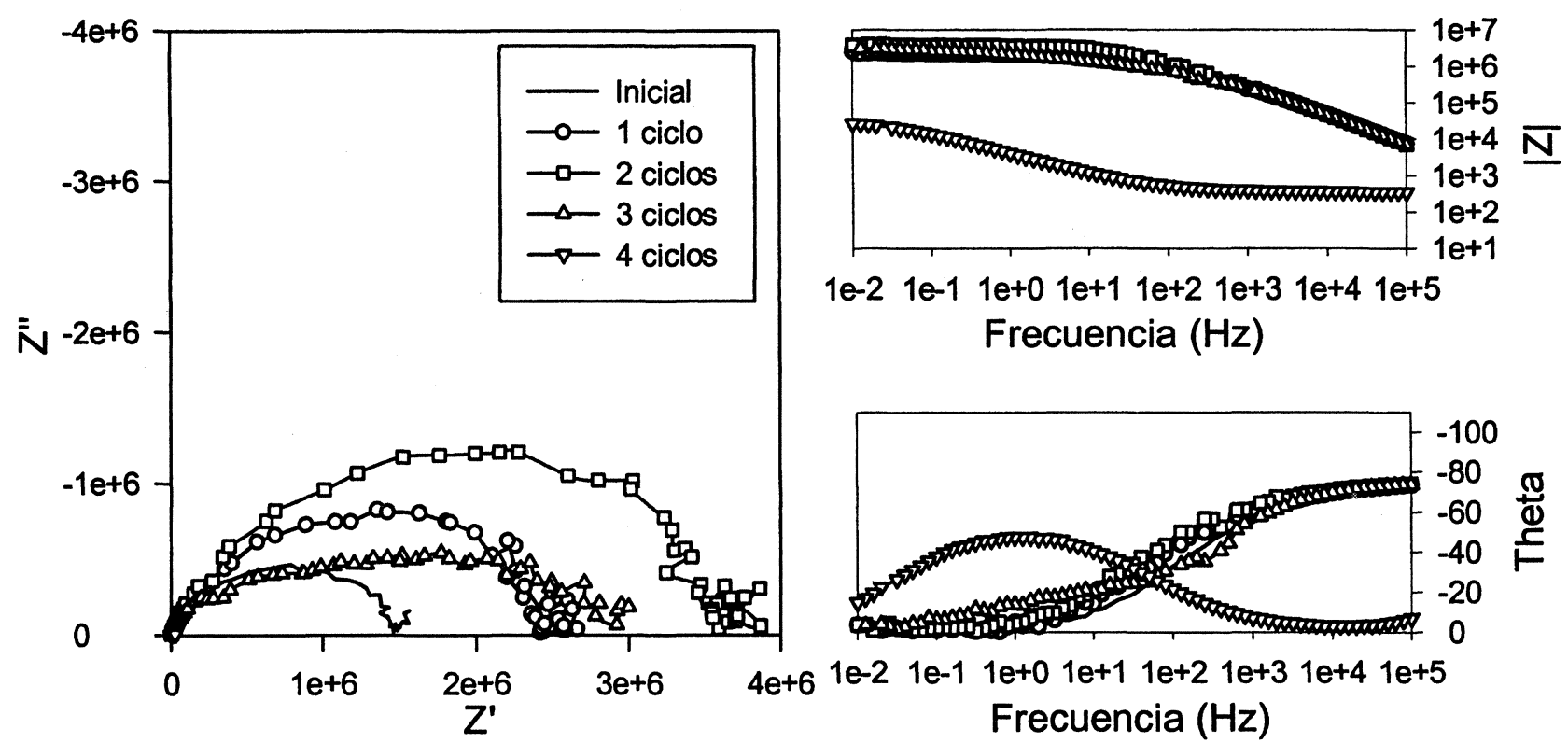

1e-2 1e-1 $1 e+01 e+1 \quad 1 e+21 e+31 e+41 e+5$ Frecuencia $(\mathrm{Hz})$

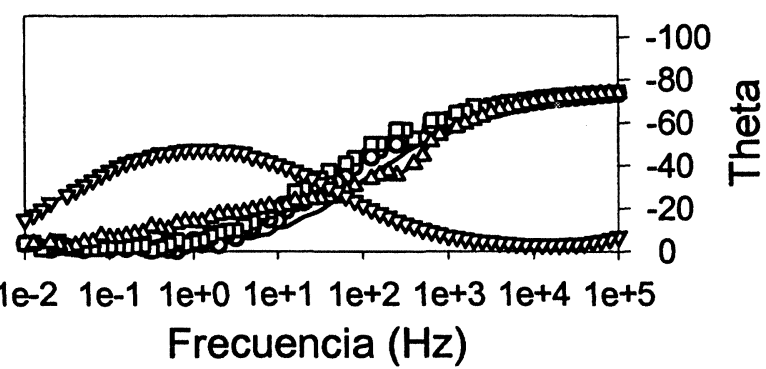

Figura 1. Espectros de EIS tras aplicar el método AC-DC-AC a la Pintura 1.

Figure 1. EIS spectra after applying cycles of AC-DC-AC method to Paint 1. 


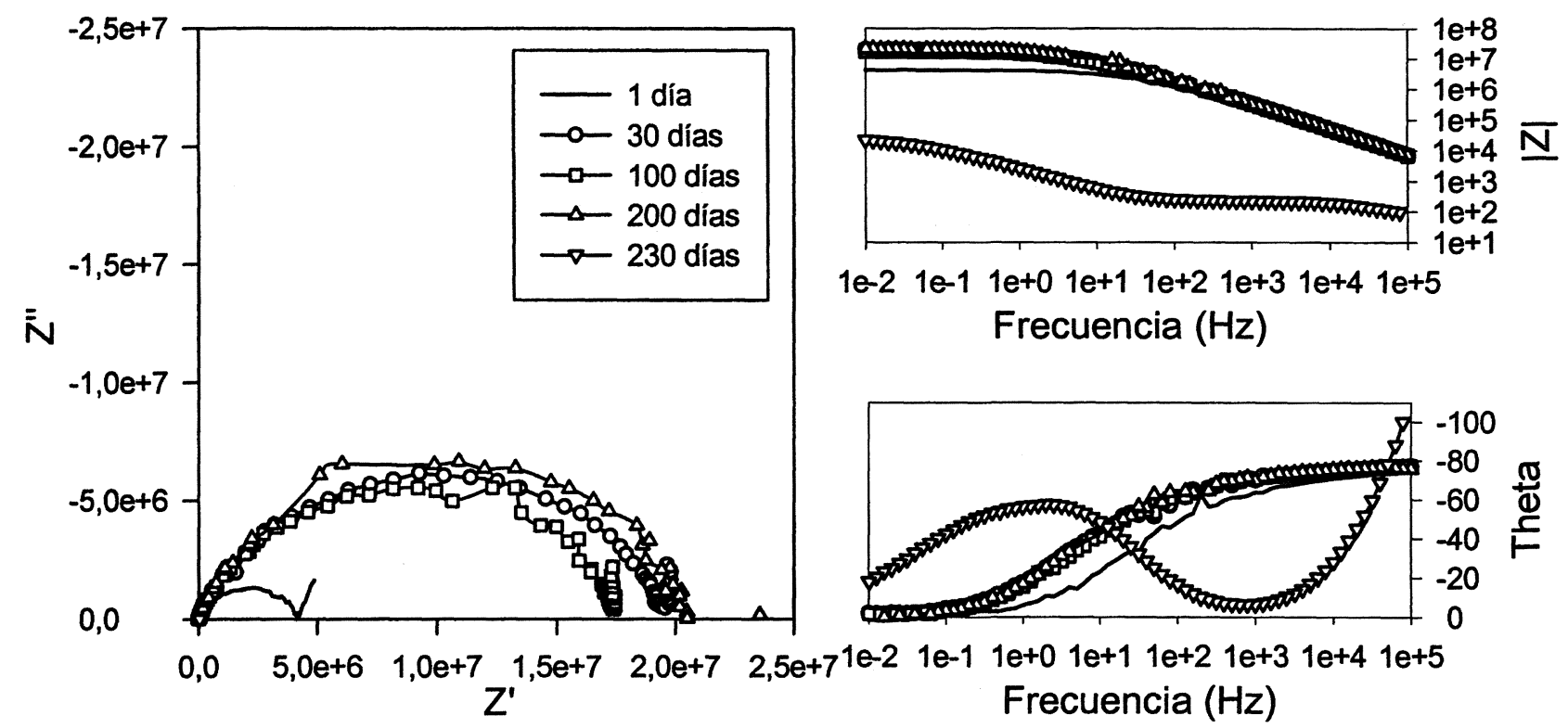

Figura 2. Evolución de EIS en el tiempo de la Pintura 1 en el medio $3.5 \% \mathrm{NaCl}$.

Figure 2. EIS evolution in time of Paint 1 immersed in a $3.5 \% \mathrm{NaCl}$ solution.
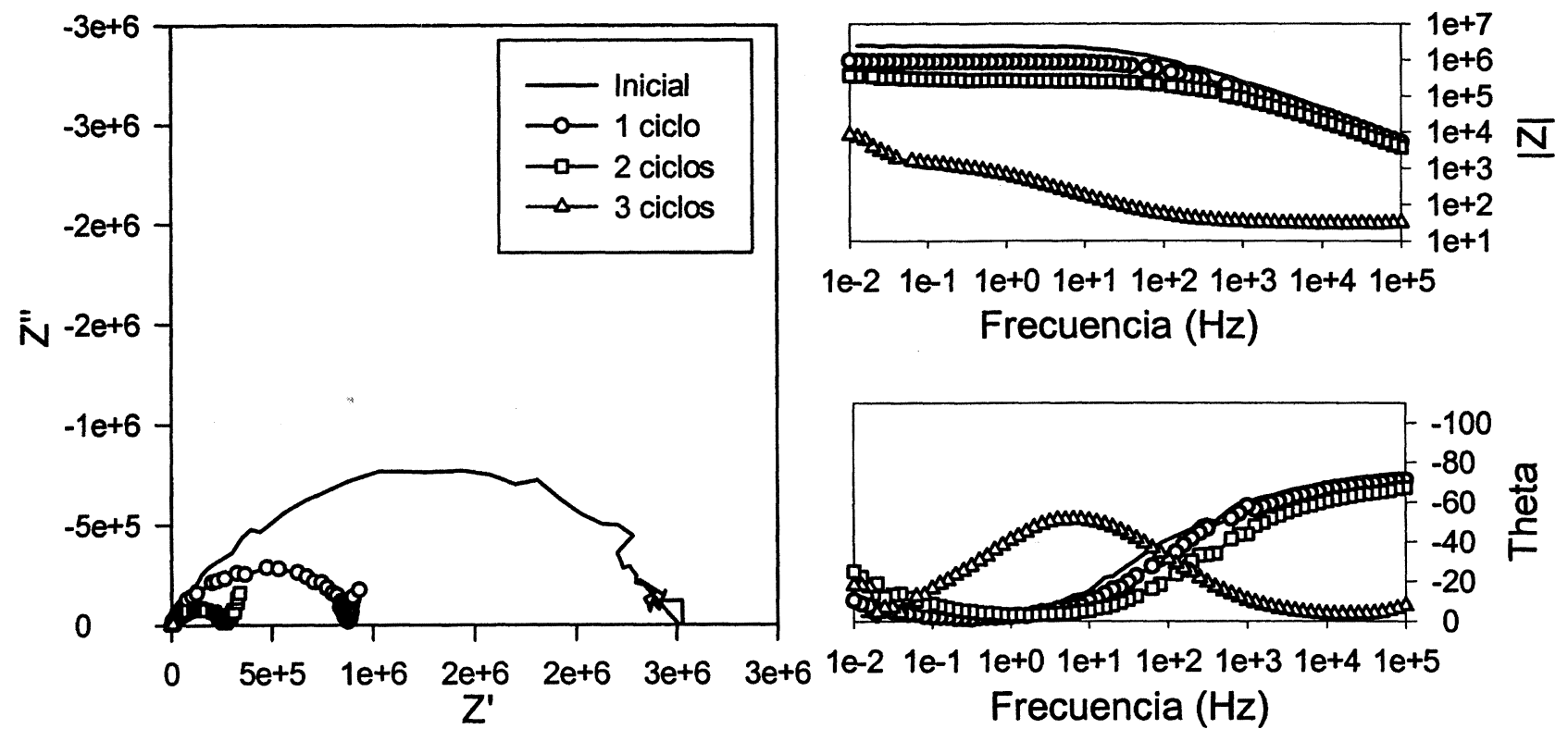

Figura 3. Espectros de EIS tras aplicar el método AC-DC-AC a la Pintura 2.

Figure 3. EIS spectra after applying cycles of AC-DC-AC method to Paint 2.

catódica. En este sentido, la degradación de aceros pintados se produce cuando el agua y el oxígeno penetran por la película de recubrimiento, se establece una capa eléctrica en la interfase metal-pintura y, finalmente, se produce la separación de la pintura respecto al metal ${ }^{[13]}$. Este proceso se denomina deslaminación catódica y se genera debido a defectos o daños mecánicos, tipo rayaduras, poros o zonas de la pintura con menor densidad de en- trecruzamiento. En estos puntos se inicia la reacción anódica u oxidación del metal, que se encuentra separada físicamente de la catódica de reducción del oxígeno, que tiene lugar en la zona adyacente. En las zonas catódicas se crea una elevada concentración de iones $\mathrm{OH}^{-}$, que elevan el $\mathrm{pH}$ del medio, provocando la pérdida de adherencia entre el metal y la pintura ${ }^{[14]}$. 

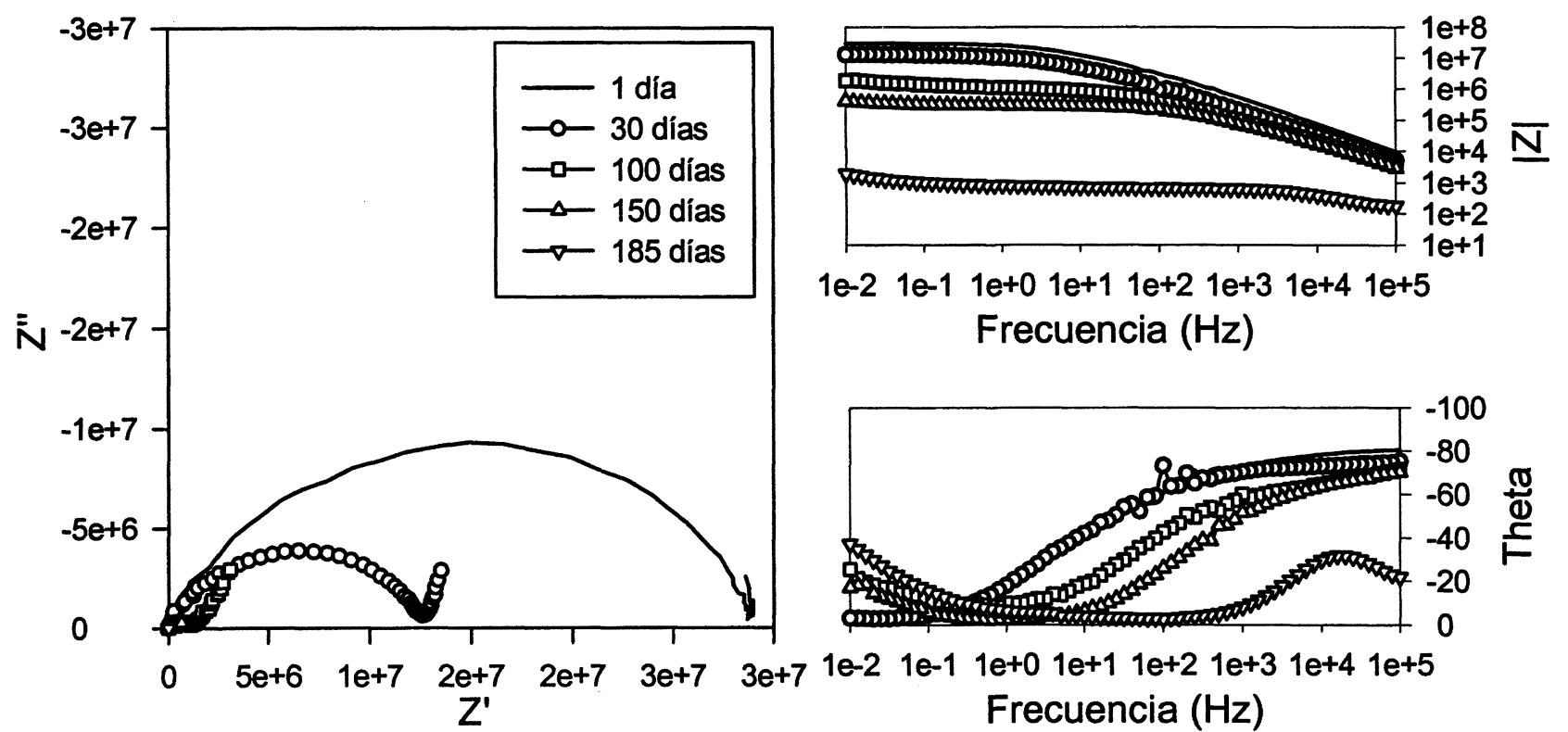

Figura 4. Evolución de EIS en el tiempo de la Pintura 2 en el medio $3.5 \% \mathrm{NaCl}$.

Figure 4. EIS evolution in time of Paint 2 immersed in a $3.5 \% \mathrm{NaCl}$ solution.

\section{CONCLUSIONES}

El método AC-DC-AC permite acelerar el proceso de evaluación de las pinturas. Asimismo, reproduce con fiabilidad el proceso de degradación observado en condiciones de exposición libre a la acción del medio corrosivo y posee la sensibilidad suficiente como para detectar cambios en las propiedades protectoras de los sistemas estudiados.

En definitiva, este método puede llegar a ser una herramienta muy útil para la evaluación rápida y clasificación de pinturas con respecto a su comportamiento anticorrosivo.

\section{Agradecimientos}

Este trabajo ha sido financiado por PETRI 95-0836 y la Comisión Interministerial de Ciencia y Tecnología, proyectos MAT2001-3477.

\section{REFERENCIAS}

[1] E. Spengler, F.L. Fragata, I.C.P. MARGARIT y O.R. MatTos, Prog. Org. Coat. 30 (1997) 51-57.

[2] D.A. Claydon. Technical Papers International Protective Coatings, Akzo Nobel, London, England, 2002, pp. 1-9.
[3] C. Pérez, A. Collazo, M. Izquierdo, P. Merino y X.R. NóvoA, Rev. Metal. Madrid 34 (1998) 122-126.

[4] N. Betancourt, F. Corvo, A. Mendoza, J. Simancas, M. Morcillo, J.A. González, F. Fragata, J.J. Peña, M. SÁnchez De Villalar, S. Flores, E. Almeida, S. Rivero y O.T. De Rincón, Rev. Metal. Madrid Vol.Extr. (2003) 38-42.

[5] D.M. Escobar, C. Arroyave, O.R. Mattos, I.C. Margarit y J. Calderón, Rev. Metal. Madrid Vol.Extr. (2003) 97-103.

[6] J. Hollaender, Food Addit. Contam. 14 (1997) 617-626.

[7] J.J. Suay, M.T. RodríGuez, R. IZQuierdo, A.H. Kudama y J.J. SAURA, J. Coat. Tech. 75 (2003) 103-104.

[8] M.T. Rodríguez, J.J. Gracenea, S.J. García, J.J. Saura y J.J. Suay, Prog. Org. Coat. 50 (2004) 123-131.

[9] M. Bethencourt, F.J. Botana, M.J. Cano, M. Marcos y R.M. Osuna, Prog. Org. Coat. 49 (2004) 275-281.

[10] C. Le Pen, C. Lacabanne y N. Pebere, Prog. Org. Coat. 46 (2003) 77-83.

[11] E. Potvin, L. Brossard y G. Larochelle, Prog. Org. Coat. 31 (1997) 363-373.

[12] B. Del Amo, R. Romagnoli, C. Deyá y J.A. González, Prog. Org. Coat. 45 (2002) 389-397.

[13] S. GonzÁlez y R.M. Souto, Materiales y Procesos Electródicos 1 (2002) 83-130.

[14] H. LEIDHEISER JR.. Corros. 38 (1982) 374-383. 